\title{
Anabases
}

ANABASES Traditions et réceptions de l'Antiquité

3 | 2006

Varia

\section{Présentation du Centre de Recherche sur la Littérature des Voyages (crlv)}

\section{François Moureau}

\section{(2) OpenEdition}

1 Journals

Édition électronique

URL : http://journals.openedition.org/anabases/2745

DOI : 10.4000/anabases. 2745

ISSN : 2256-9421

Éditeur

E.R.A.S.M.E.

\section{Édition imprimée}

Date de publication : 1 mars 2006

Pagination : 258-261

ISSN : 1774-4296

\section{Référence électronique}

François Moureau, «Présentation du Centre de Recherche sur la Littérature des Voyages (crlv)»,

Anabases [En ligne], 3 | 2006, mis en ligne le 01 janvier 2012, consulté le 20 octobre 2019. URL : http:// journals.openedition.org/anabases/2745; DOI : 10.4000/anabases.2745

Ce document a été généré automatiquement le 20 octobre 2019.

(c) Anabases 


\title{
Présentation du Centre de Recherche sur la Littérature des Voyages (crlv)
}

\author{
François Moureau
}

1 Équipe d'accueil du ministère de la Recherche, logé à l'Université de Paris-Sorbonne (Maison de la recherche, 28, rue Serpente, 75006 Paris), le CRLV est présent dans un secteur scientifique dont le développement est quasi exponentiel depuis une quinzaine d'années. Quand il fut créé en 1984 à la Sorbonne, deux équipes en France (Grenoble et ENS Fontenay-Saint-Cloud) et une en Italie (CIRVI de Turin) travaillaient de manière constante sur la littérature de voyages. En 2006, plusieurs colloques sont organisés chaque mois sur ce sujet dans les universités françaises et étrangères. À la conjonction de plusieurs domaines de recherche, la matière scientifique a suscité de nombreuses thèses au cours des dernières années. Mais le secteur n'est pas encore clairement individualisé dans les instances scientifiques et, donc, par des créations de postes. Le nombre de doctorants encadrés par le CRLV et ses partenaires montre que, dans un délai très court, ce secteur aura sa part légitime dans le spectre universitaire. Actuellement, le nombre d'enseignants chercheurs spécialisés est encore modeste. Il n'en sera plus de même, espérons-le, dans les prochaines années.

2 Institution assez ancienne, mais en perpétuel renouvellement, le CRLV est une organisation ramifiée à large ouverture scientifique et internationale. Il est structuré de manière à remplir toutes les missions d'un centre de recherche, de l'incitation scientifique à la communication de ses résultats, des colloques et séminaires aux publications imprimées et numériques. Il anime le projet BLVF (Bibliographie de la littérature de voyage française) à l'intérieur de l'UMR 8599 (Centre d'étude de la langue et de la littérature française des $\mathrm{XVII}^{\mathrm{e}}$ et $\mathrm{XVIII}^{\mathrm{e}}$ siècle, CELLF, CNRS-université ParisSorbonne) : un inventaire de la littérature primaire numérisée sur le site web du CRLV et sur celui du CELLF.

3 Inauguré en 1998, son site web (crlv.org) a une audience internationale qui en fait le point de rendez-vous des chercheurs francophones des divers continents: son 
architecture a été presque totalement reconstruite au printemps 2004, pour en faciliter la lisibilité et la consultation. En novembre 2005, il répertorie (avec bibliographie) plus de 900 chercheurs dans la spécialité, propose diverses bases de données dont nous reparlerons, une bibliographie semestrielle, des annonces, un forum ouvert, et surtout 572 conférences dans son encyclopédie sonore. En 2002, 68929 internautes différents ont fréquenté le site, 115422 en 2003, et 172410 en 2004 et plus de 215000 en 2005.

Le CRLV avait publié de 1986 à 1997 quinze volumes de la collection «Littératures des voyages», aux Éditions Klincksieck pour l'essentiel. Á la suite des déboires de notre éditeur, le directeur du CRLV a créé une nouvelle collection «Imago mundi» aux Presses de l'Université Paris-Sorbonne : douze volumes ont paru de 1999 à 2005, sur un rythme de croisière de deux à trois volumes par an, comprenant des recueils, des monographies et des éditions savantes. Plusieurs de nos collaborateurs proches y ont publié : A. Blondy, F. Lestringant, S. Linon-Chipon, F. Moureau, S. Requemora. Les autres auteurs sont des correspondants habituels du CRLV et des fidèles de ses colloques et séminaires.

5 Le séminaire annuel (25 séances) du CRLV en Sorbonne est animé par François Moureau (secrétariat: Sophie Linon-Chipon). 2000-2001: "Littérature de voyage: lieux et idéologie»; 2001-2002 : «Horizons du voyage : écrire et rêver l'univers»; 2002-2003 : « Relations savantes : voyages et discours scientifique à l'Âge classique»; 2003-2004: « Religion et sexualité dans la littérature des voyages (XVI ${ }^{\mathrm{e}}$-XVIII ${ }^{\mathrm{e}}$ siècles)»; 2004-2005 : « Civilisation et cités perdues dans la littérature des voyages (de la Renaissance au Romantisme)»; 2005-2006 : «Voyages de découverte, découverte du voyage (XVI ${ }^{\mathrm{e}}$-XVIII ${ }^{\mathrm{e}}$ siècles)». Un second séminaire annuel «Mappa mundi» a été créé en 2004 pour les études portant sur les deux derniers siècles ( Approches interdisciplinaires de la littérature de voyage des $\mathrm{XIX}^{\mathrm{e}}$ et $\mathrm{xx}^{\mathrm{e}}$ siècles»). La totalité des séminaires, auxquels participent les meilleurs spécialistes de France et de l'étranger, est disponible en version audio sur le site web du CRLV.

6 Le CRLV organise de grands colloques internationaux annuels, généralement à la Fondation Henry Clews du château de La Napoule (Alpes maritimes), institution américaine avec laquelle nous avons signé une convention renouvelée en 2004 pour quatre années. XIII Colloque, mai 2000: "L'Aventure maritime: pirates corsaires et flibustiers». XIV Colloque, mai 2001: «Récit de voyage et religion» (au Conservatoire botanique Mascarin de La Réunion); $x v^{e}$ Colloque, juin 2002: "Récits du dernier siècle des voyages». XVI Colloque, juin 2003: «Relations savantes: voyages et discours scientifique» (avec une journée au musée océanographique de Monaco. Colloque différent du séminaire). Tous ces colloques sont publiés en 2004. Outre ces colloques, le CRLV a conclu avec le château de Grignan et le Conseil général de la Drôme une convention pour trois colloques organisés à l'occasion des journées du livre d'octobre et du thème de la correspondance : ces journées «Imago mundi» ont eu lieu de 2000 à 2002 : «Lettres et images d'Europe», "Lettres et images d'ailleurs», "Le monde des cartes». Une exposition de dessins d'Hubert Robert était joint aux journées 2001 et, en 2002, une journée a été consacrée aux travaux des doctorants des universités parisiennes et du sud-est, avec prix du meilleur jeune chercheur. Les actes sont sous presse.

7 Par ailleurs, le CRLV a organisé des colloques complémentaires, seul ou en collaboration : Juin 2000 : «La Bourgogne des voyageurs» (Cuisery-Mâcon, publié). Avril 2001 : «Izmir au miroir des voyageurs français et de l'histoire» (Izmir, avec l'université 
d'Égée, publié en turc). Août 2001: «Les voyageurs européens en Chine. Images et réalités " (Shanghaï et Beijing, avec la Shanghai International Studies University sIsu et l'université de Beijing Beida). Février 2002: «Figures de pirates. Imaginaire et représentations (xvII $-\mathrm{XxI}^{\mathrm{e}}$ siècles)»(Paris, avec le musée de la Marine). Novembre 2002: «Le Livre maritime (1750-1850)» (Brest, avec l'École nationale des chartes et le Service historique de la Marine, publié). Les membres du CRLV ont participé à de nombreux autres colloques en France et à l'étranger, dont les commémorations Champlain (Québec, 2003) et Nicolas Baudin (Sydney, 2002).

8 Le CRLV a, en effet, contribué au programme canadien «Champlain 2004 » célébrant la naissance de la Nouvelle-France. Notre contribution sur l'iconographie de la NouvelleFrance était liée à l'ACI dont nous avons bénéficié en 2003. Cette ACI «Espaces et territoires», option: «Représentations», s'intitule: Littérature de voyage et iconographie : histoire et géographie des représentations viatiques des territoires, des premiers textes imprimés au début du XIX siècle (VIATICA). La durée du programme est de trente-six mois, de septembre 2003 à août 2006. Il est géré pour le Centre par Sophie Linon-Chipon, secrétaire général du CRLV. Pluridisciplinaire et international, le programme repose sur un réseau de quatre équipes francophones: le CRLV, maitre d'ouvrage, le Centre de recherche en littérature et histoire dans l'océan Indien (CRLHOI) de l'université de La Réunion, le Programme national de recherche ${ }^{\circ} 48$ (PNR48) « Paysage et habitat de l'arc alpin", rattaché au Fonds national de la recherche en Suisse et à l'université de Lausanne, le Centre interuniversitaire d'études sur les lettres, les arts et les traditions (CELAT) de l'université Laval (Québec, Qb, Canada). Cette fédération d'équipes est associée à la Commission internationale de recherche sur l'histoire des voyages et du tourisme (ICHTT), dont le siège est à l'Universita della Svizzera Italiana à Lugano et qui dépend du Comité international d'histoire des sciences (ICHS). La recherche s'inscrit dans la continuité des travaux menés depuis plusieurs années sur la notion d'imago mundi. Le site Viatica, intégré au site web du CRLV, recueille les résultats de ces enquêtes qui consistent à répertorier, dans les imprimés de voyage en langue française, de l'origine de l'imprimerie au début du dernier siècle, les illustrations qui les accompagnent. La numérisation des images est suivie d'une mise en fiche technique complète consultable sur une base de données comportant un moteur de recherche. Lors des premières journées d'études de l'ACI, en juin 2004, au château de La Napoule, la fiche technique a été discutée et mise au point en liaison avec d'autres intervenants extérieurs: Département des Estampes et de la Photographie de la Bibliothèque nationale de France, Portail documentaire du musée du Quai Branly (MQB) et Centre romantique du Cabinet Vieusseux et Maison «Iconoteca» (Florence, Italie). Des sections VIATICANADA et VIATICALPES ont été constituées. Ce programme se poursuit sans difficulté notable. 


\section{AUTEUR}

FRANÇOIS MOUREAU

françois.moureau@paris4.sorbonne.fr 